\title{
Burnout and areas of work-life among anaesthetists in South Africa Part 1: Burnout
}

\author{
JF Coetzee, ${ }^{1 D}$ H Kluyts ${ }^{2}$
}

\author{
${ }^{1}$ Department of Anaesthesiology and Critical Care, Faculty of Medicine and Health Sciences, Stellenbosch University, South Africa \\ 2 Department of Anaesthesiology, Dr George Mukhari Academic Hospital, Sefako Makgatho Health Sciences University, South Africa \\ Corresponding author, email:jfc@sun.ac.za
}

Background: Burnout is a psychological syndrome that develops in response to chronic job-related stressors. Its three predominant manifestations are emotional exhaustion, cynicism, and feelings of ineffectiveness and unfulfillment (low efficacy). Our study objectives were to establish the prevalence and severity of burnout among South African anaesthetists and to compare the results with previous studies. We also identified areas of work-life that predispose to burnout and we report these results in a separate paper (Part 2). ${ }^{1}$

Methods: We e-mailed invitations to 1852 SASA members, requesting responses to two validated questionnaires, the Maslach Burnout Inventory and the Areas of Worklife Scale. We categorised respondents according to the "Emotional Exhaustion+1" principle, whereby a person is regarded as being clinically burned-out if he/she has a a high score for emotional exhaustion, plus either a high cynicism score or a low efficacy score. High scores for all three dimensions defined "extreme burnout".

Results: 189 public sector and 309 private sector anaesthetists responded. 85\% of public sector respondents worked in academic hospitals. Compared to those in private practice, public sector anaesthetists exhibited a greater prevalence and severity of burnout. This was manifested by: (1) more adverse scores for all three burnout dimensions; (2) a greater prevalence of clinically diagnosable burnout ( $36.5 \%$ vs. $14.2 \%)$ as well as "extreme burnout" (17.5\% vs. $6.5 \%)$. Public sector anaesthetists experienced more burnout than in other countries.

Conclusions: The prevalence of burnout is unacceptably high among South African anaesthesia providers, particularly in public hospitals. This poses an immediate threat to both anaesthetists' mental and physical health and to patient safety. The severity and prevalence in teaching institutions jeopardises the current effectiveness and future sustainability of the South African healthcare system.

Keywords: burnout-professional, anaesthesiologists-psychology, job satisfaction, work engagement, physician impairment, crosssectional studies

"Never overestimate the strength of the torchbearer's arm, for even the strongest arms grow weary."

AJ Darkholme, in Rise of the Morningstar

\section{Introduction}

Burnout is a psychological syndrome that develops in response to chronic job-related stressors. ${ }^{2}$ The Royal College of Psychiatrists defines burnout as "a syndrome of emotional exhaustion, involving the development of negative self-concepts, negative job attitudes and a loss of concern and feeling for patients. Stress and burnout lead to maladaptive coping strategies". 3 Burnout is specifically related to work, and previous psychological pathology is not a prerequisite. Burnout is generally considered to comprise three dimensions, emotional exhaustion, cynicism ${ }^{+}$and (lack of) efficacy. ${ }^{\ddagger 4}$ A more detailed explanation of these three dimensions is outlined in the Appendix. The converse of burnout is engagement, a state of high energy, strong involvement, and a sense of effectiveness. ${ }^{2} \mathrm{~A}$ continuum between engagement and burnout exists. ${ }^{4}$

† Previously termed depersonalisation

‡ Previously termed personal accomplishment
Anaesthetists and intensivists rank high among physicians who suffer from burnout. ${ }^{5,6}$ A 2015 Gauteng survey ${ }^{7}$ revealed that $21 \%$ of academic hospital anaesthetists have high-risk scores for all three burnout dimensions. Their results also indicate that there may be a greater risk for burnout among academic hospital anaesthetists than those in private practice.

We investigated burnout among SA anaesthetists using a validated survey. Our primary aims were to:

1. determine the prevalence and severity of burnout among members of the South African Society of Anaesthesiologists (SASA),

2. compare our SASA sample's burnout scores with those of previous, normative studies, ${ }^{8}$ and

3. compare burnout prevalences and severity between the public and private sectors.

Secondary objectives were to compare the prevalence and severity of burnout among specific subgroups, namely between different age groups, genders, trainees and specialists, tertiary and non-tertiary hospitals, and between intensivists and anaesthetists. We also compared our South African findings with 
those of previous, overseas studies that had employed similar benchmarks for burnout.

The main hypotheses were:

1. that burnout-risk scores of SASA members exceed normative values, ${ }^{8}$ and

2. that public-sector anaesthetists exhibit a greater prevalence and severity of burnout than anaesthetists in private practice.

Secondary hypotheses were that burnout prevalence is greater among younger vs older anaesthetists, female vs male anaesthetists, intensivists vs anaesthetists, trainees vs specialists, and South Africans vs overseas-based anaesthetists. Table II summarises the primary and secondary outcomes of this study

\section{Methods}

We conducted the survey between July and September 2018. All SASA members with known e-mail addresses were invited to participate by following a link to a web-based questionnaire. The invitation also assured anonymity, requested consent, and provided investigator contact details. By responding, participants granted consent for their responses to be used in the study. Responder bias and sensitisation to burnout was limited by excluding the word "burnout" in the invitation and introducing the study as a survey of work-related attitudes.

We employed two widely-accepted, validated instruments for assessing burnout and its causes (Mind Garden Inc. Menlo Park, USA):The first instrument, the Maslach Burnout Inventory-Human Services Survey for Medical Personnel (MBI-HSS(MP)) $)^{9}$ comprises 22 items. The questionnaire evaluates the frequency and severity of the three dimensions of burnout, namely emotional exhaustion, cynicism and efficacy. Respondents indicate their frequency of work-related feelings and attitudes on a seven-point Likert-scale ranging from $0=$ never, to $6=$ every day. The second instrument, the 28-item Areas of Worklife Survey, evaluates the causes of burnout. It does this by assessing the congruence between person and work environment in six areas of work-life, namely workload, control, reward, community, fairness, and values. ${ }^{10-13} \mathrm{We}$ report our findings regarding the Areas of Worklife separately, in Part 2 of this study. ${ }^{1}$

The survey also requested social and demographic data including gender, age group, years since qualification, public or private practice, and level of qualification (specialist, trainee, practitioner holding a Diploma of Anaesthesia, or general practitioner).
Completed questionnaires were automatically captured and stored on a secure REDCap server (https://projectredcap.org/).

Sample size was calculated using Equation A4 (Appendix). A 4\% margin of error, with $95 \%$ confidence level and correction for a population of 1852 potential responders, required a sample of 454. We aimed to recruit 500 or more anaesthesiologists.

\section{Data analysis}

We downloaded our data from the REDCap database into an Excel spreadsheet for analysis. Using the burnout categorisation scores depicted in Table l, we categorised participants' scores for emotional exhaustion, cynicism, and efficacy as high, moderate, or low.

The authors of the Maslach Burnout Inventory (MBI) emphasise that each of the three burnout dimensions should be evaluated separately, and that combining the scores into a single grade is invalid..$^{9}$ However, for clinical purposes there is a need for a method by which to arrive at a dichotomous, clinical diagnosis of burnout, especially considering that in several European countries, burnout warrants sick leave (ICD-10 code Z73.0). Brenninkmeijer and Van Yperen proposed the "Emotional Exhaustion +1 " principle, whereby a person can be diagnosed as being clinically burned out if he/she has a high score for emotional exhaustion plus either a high cynicism score or a low efficacy score. ${ }^{14}$ The authors of the Maslach Burnout Inventory concur that the "Emotional Exhaustion +1 " rule ${ }^{14}$ defines a psychological state of sufficient severity that justifies a clinical diagnosis of burnout. 15,16 We therefore analysed our questionnaire score results using a dual approach: (1) an analysis of the three burnout dimensions separately, and (2) an analysis of the proportions of respondents who could be diagnosed as being clinically burned out according to the "Emotional Exhaustion+1" rule. In addition we defined "extreme burnout" as high scores for all three burnout dimensions, which is a diagnosis that has been used by nine previous studies. ${ }^{17}$

To compare our findings with those from previous studies regarding burnout among doctors, we searched for studies that used both the complete Maslach Burnout Inventory ${ }^{\ddagger}$ as well as similar burnout definitions. Rotenstein and co-workers attempted a meta-analysis of 182 studies that included 109628 physicians from 45 countries. ${ }^{17}$ Sanfilippo and colleagues attempted a meta-analysis of burnout studies that investigated only anaesthesiologists. ${ }^{18}$ Both groups were unable to complete their intended meta-analyses because of cut-off point and score classification heterogeneity. Nonetheless, these papers allowed us to identify several studies that met our criteria for comparative

¥ As opposed to a shortened version of the Maslach Burnout Inventory.

Table I: Categorisation of burnout scores

\begin{tabular}{|c|c|c|c|c|}
\hline \multirow{2}{*}{$\begin{array}{l}\text { Burnout } \\
\text { dimension }\end{array}$} & \multirow{2}{*}{ Range } & \multicolumn{3}{|c|}{ Score categories } \\
\hline & & High & Moderate & Low \\
\hline Emotional exhaustion & $0-63$ & $>26$ & $19-26$ & $0-18$ \\
\hline Cynicism & $0-35$ & $>9$ & $6-9$ & $0-5$ \\
\hline Efficacy & $0-56$ & $>39$ & $34-39$ & $0-33$ \\
\hline
\end{tabular}

Note that a low score for efficacy indicates high risk for burnout 


\begin{tabular}{|c|c|}
\hline \multicolumn{2}{|r|}{ Primary outcomes } \\
\hline Variables & Analysis \\
\hline $\begin{array}{l}\text { Maslach Burnout Inventory scores for } \\
\text { emotional exhaustion, cynicism and } \\
\text { efficacy considered separately }\end{array}$ & $\begin{array}{l}\text { 1. Compare the scores of whole SASA sample with scores from a normative study }{ }^{9} \\
\text { 2. Comparisons between public and private sectors } \\
\text { - Compare scores for each burnout dimension } \\
\text { - Compare proportions of participants returning high and low scores for each burnout dimension }\end{array}$ \\
\hline $\begin{array}{l}\text { Prevalences of "clinical" and "extreme" } \\
\text { burnout }\end{array}$ & $\begin{array}{l}\text { 1. Establish prevalences in the sample of SASA respondents taken as a whole } \\
\text { 2. Compare prevalences between public and private sector anaesthetists }\end{array}$ \\
\hline \multicolumn{2}{|r|}{ Secondary outcomes } \\
\hline $\begin{array}{l}\text { 1. Compare prevalences of clinically diagnos } \\
\text { 2. Compare the SASA results with those in } p\end{array}$ & $\begin{array}{l}\text { sable burnout among subgroups } \\
\text { orevious, overseas studies } 17,19-24\end{array}$ \\
\hline
\end{tabular}

purposes. We independently identified two other studies of burnout among anaesthetists that employed the "Emotional Exhaustion +1 " diagnostic principle. ${ }^{19,20}$ We compared information from all identified studies with our findings.

\section{Statistical analysis}

Table II summarises the primary and secondary outcomes of this study.

Internal consistency of the questionnaire was estimated by calculating Cronbach's alpha coefficient. ${ }^{25,26}$ Using two-sided t-tests, we compared mean scores of the entire sample for the three burnout dimensions with those from a pre-identified normative study involving 1104 healthcare workers. ${ }^{8}$ As within study group, data were not normally distributed; we compared the data using the Mann-Whitney $U$ test for unpaired data and the Wilcoxon signed rank test for paired data. An alpha value $<0.05$ was accepted as indicating a significant difference. We calculated ninety five percent confidence intervals $(95 \% \mathrm{Cl}$ ) for differences between medians according to the HodgesLehmann method. ${ }^{27,28}$ We compared proportional data using the chi-square test. We performed the Marascuilo procedure for nonparametric post hoc pairwise comparisons when analysing contingency tables that were larger than $2 \times 2 .{ }^{29,30}$ We calculated 95\% Cl for proportions according to Wilson's method. ${ }^{27}$ Survey scores derived from Likert scales are not intuitively meaningful clinical measurements. Therefore, differences between means, medians, proportions and their $95 \% \mathrm{Cls}$ are difficult to interpret. In order to add meaning to the differences between groups, we report appropriate measures of effect-size, namely relative risk for differences between proportions and probability of superiority for the Mann-Whitney $U$ and Wilcoxon signed ranks tests $^{31}$ (Tables A1 and A2, Appendix). The statistical software we employed was MedCalc Statistical Software (Ostend, Belgium) and Confidence Interval Analysis (T Bryant). ${ }^{32,33}$

\section{Results}

This paper reports the results of the burnout, questionnaire (MBI-HSS(MP)). We report the Areas of Worklife Survey in a separate paper. ${ }^{1}$ There were 498 completed questionnaires: this represented a $26.9 \%$ response rate and carried a $3.76 \%$ margin of error. The Cronbach's alpha coefficient (95\% lower confidence limit) was 0.92 (0.91) for the questionnaire. For the three burnout dimensions, the alpha coefficients were 0.93 (0.92), $0.78(0.75)$ and $0.76(0.73)$ for emotional exhaustion, cynicism and efficacy respectively. These coefficients exceed the requisite reliability thresholds for research..$^{25}$

The majority of respondents were male (53\%) specialists (76\%) and in private practice (74\%), of whom $57 \%$ were part of a group practice. Public sector respondents worked predominantly (84\%) in academic/tertiary hospitals. Eight intensivists responded (1.6\%) (Table V).

Table III displays the mean scores of the respondents taken as a whole. The mean emotional exhaustion score of all 498 participants was significantly lower than normative values, with a small effect-size. Differences from mean, normative values regarding cynicism and efficacy were not significant, with small effect-sizes. Overall 113 of the 498 respondents (22.7\%) were clinically burned out according to the "Emotional Exhaustion +1 " principle. Of these, 37 (7.4\%) had high emotional exhaustion +

Table III: Comparison of mean burnout scores returned by 498 SASA respondents, with normative values ${ }^{8}$

\begin{tabular}{|c|c|c|c|c|c|c|}
\hline Burnout dimension & & Mean & SD & 95\% $\mathrm{Cl}$ difference & $p$ & Effect size (Glass's $\boldsymbol{\Delta}$ ) \\
\hline \multirow{2}{*}{ Emotional exhaustion } & SASA & 21.0 & 12.4 & \multirow{2}{*}{0.8 to 3.0} & \multirow{2}{*}{0.0008} & \multirow{2}{*}{$\begin{array}{c}0.15 \\
\text { (small) }\end{array}$} \\
\hline & Normative & 22.9 & 9.53 & & & \\
\hline \multirow{2}{*}{ Cynicism } & SASA & 6.8 & 5.9 & \multirow{2}{*}{-0.3 to 0.9} & \multirow{2}{*}{0.339} & \multirow{2}{*}{$\begin{array}{c}0.054 \\
\text { (very small) }\end{array}$} \\
\hline & Normative & 7.1 & 5.2 & & & \\
\hline \multirow{2}{*}{ Efficacy } & SASA & 36.5 & 7.6 & \multirow{2}{*}{-0.7 to 0.9} & \multirow{2}{*}{0.842} & \multirow{2}{*}{$\begin{array}{c}0.011 \\
\text { (very small) }\end{array}$} \\
\hline & Normative & 36.5 & 7.3 & & & \\
\hline
\end{tabular}

SASA - SASA respondents' scores; Normative - normative scores ${ }^{8}$

SD - standard deviation; $95 \% \mathrm{Cl}$ difference $-95 \%$ confidence interval of the difference between the mean values

Interpretation of Glass's $\Delta: 0=$ no effect; $0.2=$ small effect; $0.5=$ medium effect; $0.8=$ large effect. For the calculation of Glass's $\Delta$, the denominator was the standard deviation of the normative (control) group. 
Table IV: Comparisons of the burnout scores between SASA members working in the public $(n=189)$ and the private sector $(n=309)$

\begin{tabular}{|c|c|c|c|c|c|c|c|c|}
\hline & Sector & Median score & Score category & Interquartile range & Range & 95\% $\mathrm{Cl}$ difference & $p$ & Effect size $^{\dagger}$ \\
\hline \multirow{2}{*}{$\begin{array}{l}\text { Emotional } \\
\text { exhaustion }\end{array}$} & Public & 26 & Moderate & $16.8-35.0$ & $1-54$ & \multirow{2}{*}{6 to 11} & \multirow{2}{*}{$<0.0001$} & \multirow{2}{*}{$\begin{array}{c}0.69 \\
\text { (medium) }\end{array}$} \\
\hline & Private & 15 & Low & $9.8-25.0$ & $0-52$ & & & \\
\hline \multirow{2}{*}{ Cynicism } & Public & 7 & Moderate & $4-12$ & $0-29$ & \multirow{2}{*}{2 to 4} & \multirow{2}{*}{$<0.0001$} & \multirow{2}{*}{$\begin{array}{c}0.66 \\
\text { (medium) }\end{array}$} \\
\hline & Private & 4 & Low & $2-8$ & $0-26$ & & & \\
\hline \multirow{2}{*}{ Efficacy } & Public & 35 & Moderate & $30-40$ & $1-48$ & \multirow{2}{*}{2 to 4} & \multirow{2}{*}{$<0.0001$} & \multirow{2}{*}{$\begin{array}{c}0.62 \\
\text { (small) }\end{array}$} \\
\hline & Private & 39 & Moderate & $32.8-43.3$ & $15-48$ & & & \\
\hline
\end{tabular}

95\% Cl difference - 95\% confidence interval of the difference between medians

$p$ - $p$-value generated by Mann-Whitney $\mathrm{U}$ test

† Probability of superiority. Interpretation: $0.5=$ No effect; $0.56=$ small effect; $0.64=$ medium effect; $0.71=$ large effect; $0.8=$ very large effect.

Note that higher scores for emotional exhaustion and cynicism, and lower scores for efficacy indicate increasing risk of burnout

high cynicism scores, 23 (4.6\%) had high emotional exhaustion + low efficacy scores, and 53 (10.6\%) suffered "extreme burnout" (high scores for all three burnout dimensions).

Comparisons between scores for the 3 burnout dimensions in public sector anaesthetists versus those in private practice, are shown in Table IV and in Figure 1. Anaesthetists in the public sector had significantly higher median emotional exhaustion and cynicism scores than those in the private sector $(p<0.0001)$. The median efficacy score was significantly lower in anaesthetists in the public sector $(p<0.0001)$, indicating greater adversity*. Effect-sizes were medium for emotional exhaustion and cynicism, and small for efficacy.

With regard to high, moderate, and low score categories, greater proportions of public sector anaesthetists returned high scores for emotional exhaustion and cynicism and low scores for efficacy. Associated relative risks were high (Figure 1). Accordingly, smaller proportions of public sector anaesthetists had low scores for emotional exhaustion and cynicism and high scores for efficacy (indicators of engagement).

A comparison between the prevalence of clinically diagnosable burnout in public sector anaesthetists versus those in private practice, is shown in Table $\mathrm{V}$ and Figure 2. Both clinically diagnosable burnout and "extreme" burnout were more prevalent in the public than the private sector, with high associated relative risks.

Subgroup comparisons regarding the prevalence of clinically diagnosable burnout are shown in Table V. Within the public sector, there were no significant proportional differences between tertiary and non-tertiary hospitals, or between trainees and specialists. In the private sector, there were no significant differences between solo, partnership or associationtype practices. We studied the influence of age on burnout. Respondents aged $>65$ years had the smallest proportion of clinically burned out members (7\%), which was significantly different from the age-groups $23-34$ years (37\%) and 35-44 years (24\%). We also studied the effect of experience on burnout. Those with > 15 years' experience had a significantly smaller proportion of burned-out members. Three of eight intensivists

* High scores for emotional exhaustion and cynicism and a low score for efficacy indicate high-risk for the burnout syndrome.

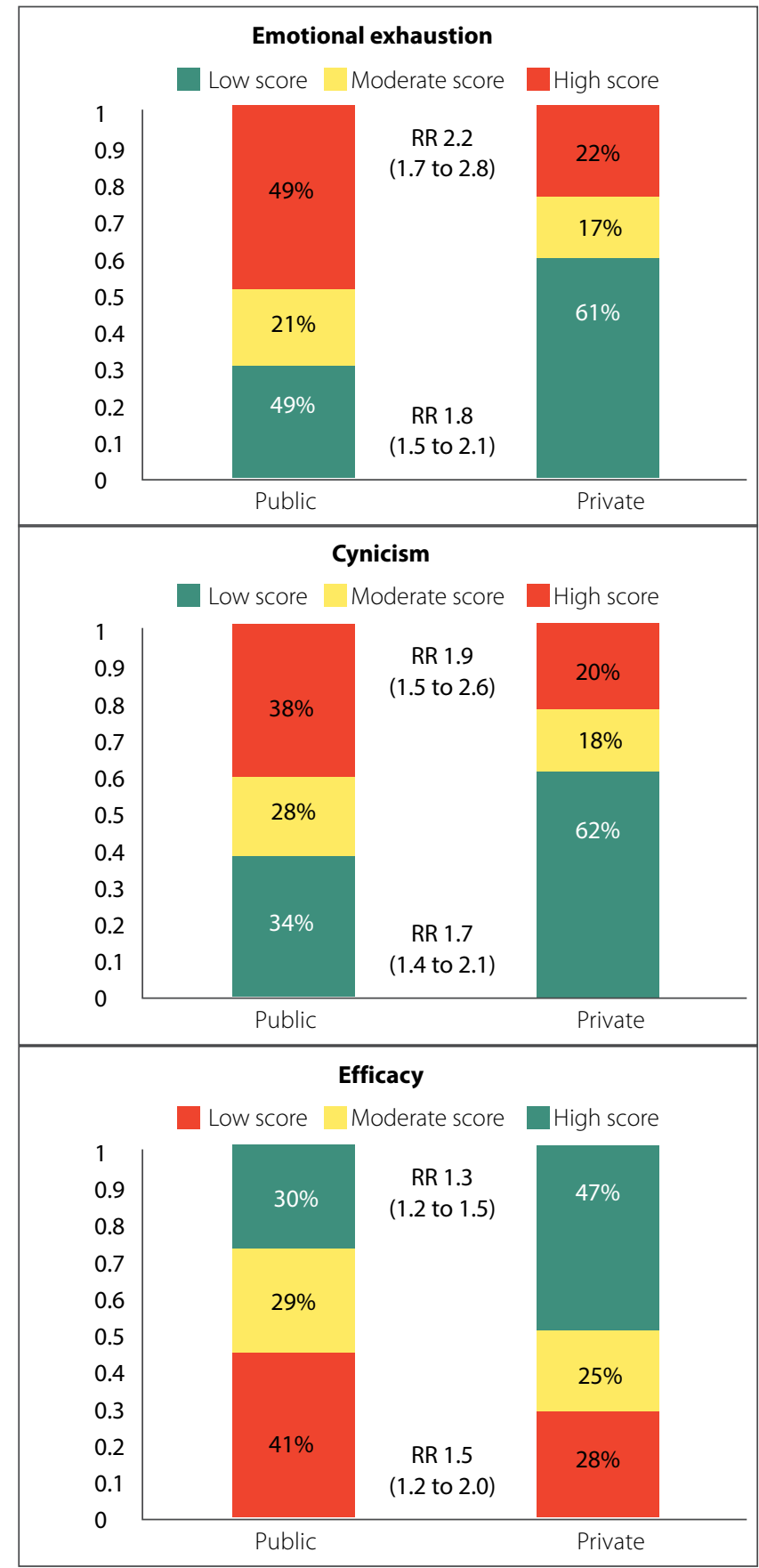

Figure 1: Proportions of participants in the public and private sector who returned high, moderate and low scores for the three burnout dimensions

Colour codes indicate risk categories for burnout: Red - high risk; Yellow - moderate risk Green - low risk. RR - relative risk ( $95 \%$ confidence interval) Note that lower scores for efficacy indicate higher risk of burnout (therefore coloured red) 


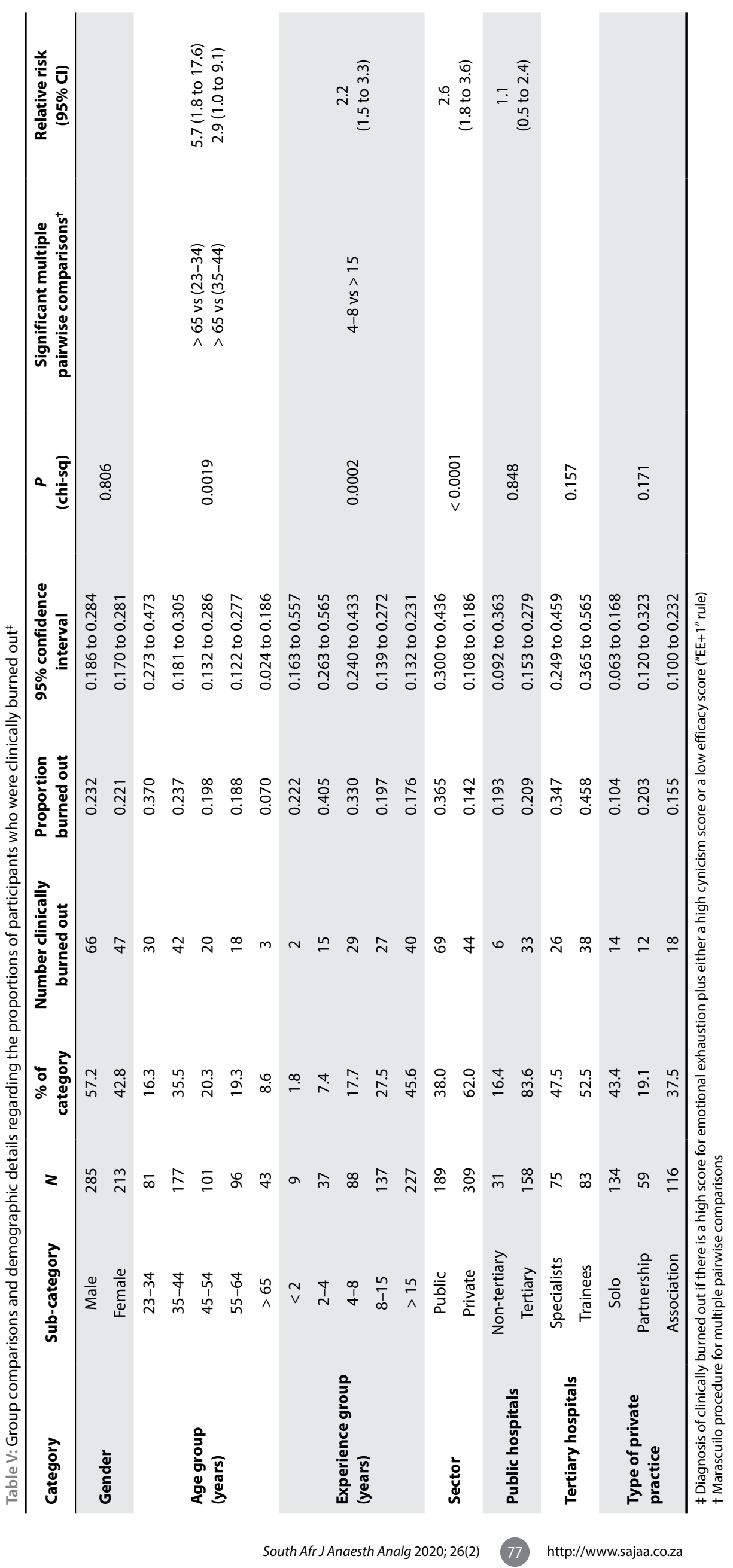



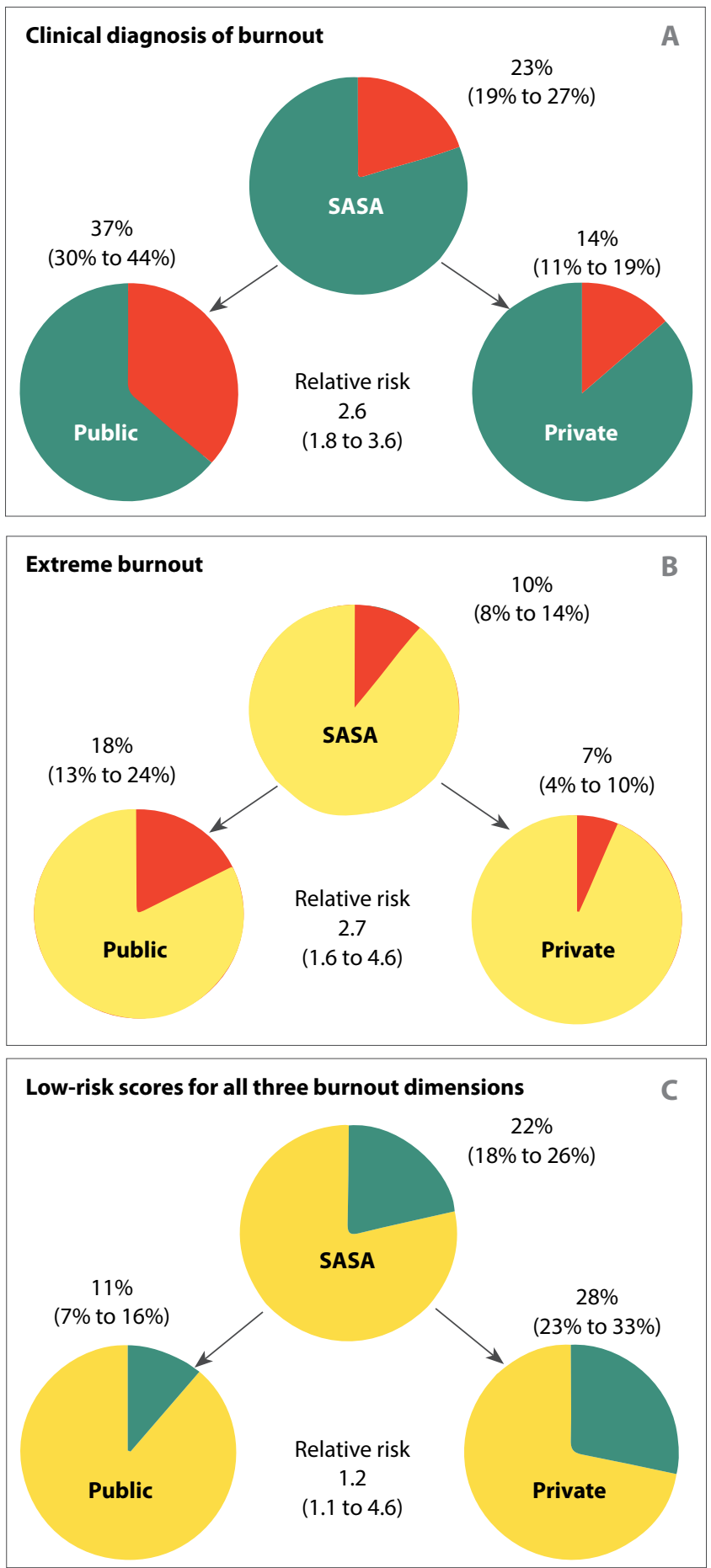

Figure 2: Proportions of anaesthetists with clinical burnout or engagement

A: Proportions of anaesthetists who could be diagnosed as being clinically burned out according to the "Emotional Exhaustion plus one" rule: i.e. a high score for emotional exhaustion plus a high score for cynicism or a low score for efficacy B: Proportions of anaesthetists who suffered "extreme burnout": i.e. a high score for emotional exhaustion plus a high score for cynicism plus a low score for efficacy $\mathrm{C}$ : Proportions of anaesthetists with low-risk scores for all three dimensions of burnout and who can be regarded as having high levels of engagement with their work

were clinically burned-out; however, the group was too small for statistical analysis (prevalence $37.5 \% ; 95 \% \mathrm{Cl} 13.7 \%$ to $69.4 \%$ ).

Comparisons with previous studies are shown in Table VI. Using the information drawn from previous studies, we compared prevalences of high-risk scores for burnout between SASA members and those of overseas studies. We also compared prevalences of clinically diagnosable burnout and "extreme" burnout. Prevalences among South African anaesthetists in private practice were either smaller or did not differ significantly from those in overseas doctors. South African public sector anaesthetists consistently revealed higher-risk scores for all three burnout dimensions, as well as greater prevalences of clinically diagnosable burnout and "extreme burnout".

\section{Discussion}

Overall, regarding the 498 SASA respondents, we did not detect significant differences from normative scores for the cynicism and efficacy burnout dimensions. Mean emotional exhaustion was significantly lower than the normative score, however the effect size was small. Thus, our first hypothesis that burnout scores of SASA members would exceed normative values was not confirmed. However, considering the overall $22.7 \%$ prevalence of clinically diagnosable burnout, it would be misleading to conclude that all is well amongst South African anaesthetists. Even more concerning is the confirmation of our second hypothesis, namely that the prevalence and severity of burnout among public sector anaesthetists exceeded that of private practitioners. The plight of public sector anaesthetists is highlighted by their $36.5 \%$ prevalence of clinical burnout. This reflects the large proportions of these respondents with adverse scores for the three burnout dimensions (Figure 1). Additionally, the prevalence of "severe" burnout was $17.5 \%$ in the public sector vs $6.5 \%$ in the private sector. The predicament of SA public sector anaesthetists is further underscored by evidence of greater burnout in this group than is to be found in several other countries (Table VI).

Our results agree with those of the 2015 study by Van der Walt and coworkers, ${ }^{7}$ whose data indicate that there was a greater proportion of academic anaesthetists with high scores for emotional exhaustion and cynicism compared with Gauteng private practitioners; relative risk $(95 \% \mathrm{Cl})$ : emotional exhaustion 2.8 (1.8 to 4.4); cynicism 1.9 (1.3 to 2.8). Our findings also concur with those of Sirsawy and co-workers who surveyed 205 Bloemfontein anaesthesia registrars and medical officers. ${ }^{34}$ Using slightly different cut-offs for the MBI-HSS(MP) scales, they concluded that $15.6 \%$ had high-risk scores for all three burnout dimensions, and that $42.0 \%$ were clinically burned out.

Healthcare worker burnout has negative consequences for quality of care, personnel health, and healthcare organisations. ${ }^{35-37}$ Burned out healthcare workers experience negative feelings towards patients, ${ }^{37}$ deliver poorer quality of care and make more errors, ${ }^{38-43}$ resulting in increasing malpractice claims. ${ }^{44}$ These issues are particularly relevant to anaesthesia. In order to mitigate risk and enhance perioperative patient safety, anaesthetists should ideally have high levels of engagement. Our study suggests that such levels of engagement are not always present among South African anaesthetists, as only $11 \%$ of public and $28 \%$ of private practitioners returned low scores for all three burnout dimensions. Furthermore, our study indicates that about one of every five private practitioner anaesthetists and 


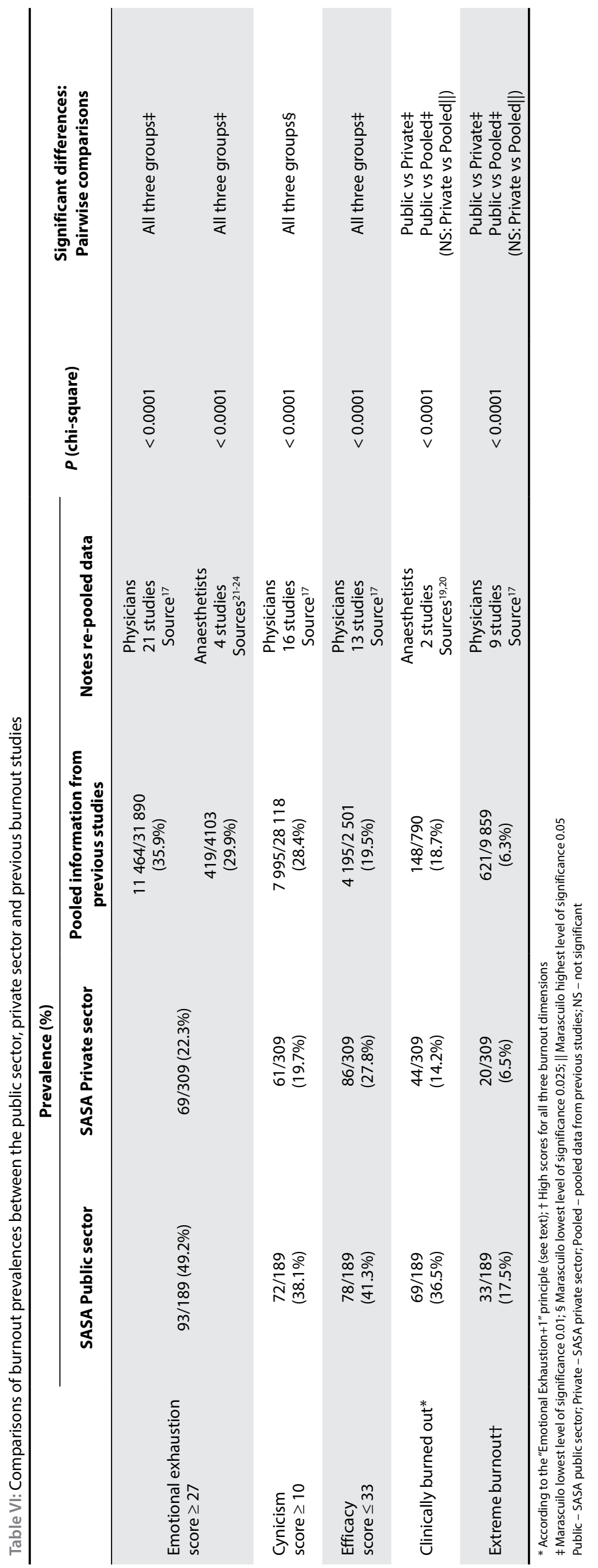

one in three academic anaesthetists can be diagnosed with clinical burnout.

Burnout also contributes to poor health among workers, ${ }^{45-52}$ poor self-care and interpersonal aggression..$^{53}$ Burnout can be contagious within a department. ${ }^{54-56}$ Despite its non-inclusion in the Diagnostic and Statistical Manual of Mental Disorders (DSM-5), several authorities on the topic assert that burnout is actually a depressive disorder. $38,57-61$

Healthcare organisations eventually suffer ${ }^{62}$ from decreased efficiency, increased absenteeism, ${ }^{52,63,64}$ and high staff turnover. ${ }^{36}$ The current high prevalence of burnout among academic hospital specialists may have widespread adverse consequences for future healthcare in South Africa. This small contingent is responsible for training future specialist anaesthetists. These specialists are perhaps not coping with the concurrent demands of onerous clinical and administrative workloads, teaching obligations, and supervision of trainee research projects. Furthermore, sufficient numbers of well-trained anaesthetists are essential to healthcare systems considering the multitude of their critical functions. Besides caring for patients in operating rooms, anaesthetists play vital roles in perioperative medicine (including preoperative evaluation and optimisation, and postoperative care). Additionally, anaesthetists are involved in intensive care, acute and chronic pain management, acute resuscitation and airway management, and procedural sedation (e.g. for endoscopic procedures, radiology, cardiology, paediatric oncology, and psychiatry). An overstressed, dysfunctional anaesthesia service will surely result in widespread deleterious effects throughout a healthcare organisation.

A limitation of this study is the low $29.6 \%$ response rate. Nevertheless, the reliability and consistency are good as indicated by the low $<4 \%$ margin of error and the adequate Cronbach's alpha coefficients. Bias is an inherent limitation of all burnout surveys. This stems from burnout sufferers being either more inclined to participate or shun e-mail requests to complete questionnaires.

We conclude that the prevalence of the burnout syndrome is unacceptably high among South African anaesthesia providers, particularly in public hospitals. This poses an immediate threat to the mental and physical health of anaesthetists, and to the quality of patient care. The severity and prevalence of public teaching sector burnout is a real threat to the current effectiveness and future sustainability of the South African healthcare system.

\section{Acknowledgements}

Mr Dawid van Straaten for transcribing the questionnaires to the REDCap system and for his persistence in spite of an erratic email server. 
Professor Andrew Levin for his helpful improvements to the manuscript text.

\section{Conflict of interest}

The authors declare no conflict of interest.

\section{Funding source}

The SASA Jan Pretorius Research Fund funded licences for the questionnaires.

\section{Ethics approval}

The Stellenbosch University Research Ethics Committee, Humanities, granted approval to conduct this anonymised, prospective, analytical, cross-sectional study (Project No.6254). SASA Council granted permission to access their membership list.

\section{ORCID}

\section{JF Coetzee (iD https://orcid.org/0000-0002-9925-7767} H Kluyts (iD) https://orcid.org/0000-0002-9917-1330

\section{References}

1. Coetzee JF, Kluyts $H$. Burnout and areas of work-life among anaesthetists in South Africa. Part 2: Areas of work-life. Southern African J Anaesth Analg 2020;26(2):83-90. https://doi.org/10.36303/SAJAA.2020.26.2.2359.

1. Maslach C, Leiter MP. Understanding the burnout experience: recent research and its implications for psychiatry. World Psychiatry. 2016 Jun;15(2):103-11. https://doi.org/10.1002/wps.20311.

2. Royal College of Psychiatrists. PSS information guide: looking after yourself. Psychiatrists Support Service [serial on the Internet]. 2019: Available from: https://www.rcpsych.ac.uk/docs/default-source/members/supporting-you/pss/ pss-15-looking-after-yourself.pdf?sfvrsn=1058bfbf_2.

3. Maslach C, Leiter MP. Burnout. In: Fink G, editor. Encyclopedia of Stress. 2nd ed: Elsevier; 2007. p. 368-71. https://doi.org/10.1016/B978-012373947-6.00062-3.

4. Neto GFD. Occupational well-being in anesthesiologists. Rio de Jeneiro: Brazilian Society of Anesthesiology; 2017.

5. Peckham C. Medscape critical care lifestyle report 2016: bias and burnout Medscape News \& Perspective [serial on the Internet]. 2016: Available from: https://www.medscape.com/features/slideshow/lifestyle/2016/ critical-care\#page $=1$

6. Van der Walt N, Scribante J, Perrie H. Burnout among anaesthetists in South Africa. Southern African Journal of Anaesthesia and Analgesia. 2015 Nov 2, 2015;21(6):169-72. https://doi.org/10.1080/22201181.2015.1102798

7. Maslach C, Jackson S, Leiter MP. Maslach Burnout Inventory Manual. 3rd ed. Menlo Park, CA: Mind Garden Inc.; 2010.

8. Maslach $C$, Jackson S, Leiter MP. Maslach Burnout Inventory Manual. 4th ed. Menlo Park, CA: Mind Garden Inc.; 2018.

9. Leiter MP, Maslach C. Areas of Worklife Survey Manual and Sampler Set. 5th ed. Menlo Park, CA: Mind Garden Inc.; 2011. https://doi.org/10.1037/t06444-000.

10. Maslach C, Leiter MP. The truth about burnout: How organizations cause stress and what to do about it. San Francisco: John Wiley \& Sons; 1997.

11. Leiter MP, Maslach C. Six areas of worklife: a model of the organizational context of burnout. J Health Hum Serv Adm. 1999 Spring:21(4):472-89.

12. Gregory ST, Menser T. Burnout among primary care physicians: A test of the Areas of Worklife model. J Healthc Manag. 2015 Mar-Apr;60(2):133-48.

13. Brenninkmeijer $V$, VanYperen $N$. How to conduct research on burnout: advantages and disadvantages of a unidimensional approach in burnout research. Occup Environ Med. 2003 Jun;60 Suppl 1:i16-20. https://doi. org/10.1136/oem.60.suppl_1.i16.

14. Maslach C, Leiter MP, Schaufeli WB. Measuring burnout. In: Cartwright S, Cooper $\mathrm{CL}$, editors. The Oxford handbook of organizational well-being. Oxford: Oxford University Press; 2009. p. 86-108. https://doi.org/10.1093/oxfordhb/9780199211 913.003.0005

15. Schaufeli WB, Leiter MP, Maslach C. Burnout: 35 years of research and practice. Career Development International. 2009;14(3):204-20. https://doi. org/10.1108/13620430910966406.

16. Rotenstein LS, Torre $M$, Ramos $M A$, et al. Prevalence of burnout among physicians: a systematic review. JAMA. 2018 Sep 18;320(11):1131-50. https://doi. org/10.1001/jama.2018.12777.

17. Sanfilippo F, Noto A, Foresta $G$, et al. Incidence and factors associated with burnout in anesthesiology: a systematic review. Biomed Res Int. 2017;2017:8648925. https://doi.org/10.1155/2017/8648925.

18. Capuzzo M, Gilli G, Paparella $L$, et al. Factors predictive of patient satisfaction with anesthesia. Anesth Analg. 2007 Aug;105(2):435-42. https://doi. org/10.1213/01.ane.0000270208.99982.88.
19. Van der Wal RA, Bucx MJ, Hendriks JC, Scheffer GJ, Prins JB. Psychological distress, burnout and personality traits in Dutch anaesthesiologists: A survey. Eur J Anaesthesiol. 2016 Mar:33(3):179-86. https://doi.org/10.1097/ EJA.00000000000000375.

20. Kluger MT, Townend K, Laidlaw T. Job satisfaction, stress and burnout in Australian specialist anaesthetists. Anaesthesia. 2003 Apr;58(4):339-45. https:// doi.org/10.1046/j.1365-2044.2003.03085.x

21. Magalhães E, Oliveira ÁC, Govêia CS, et al. Prevalence of burnout syndrome among anesthesiologists in the Federal District. Rev Bras Anestesiol. 2015;65(2):104-10. https://doi.org/10.1016/j.bjan.2013.07.016.

22. Morais A, Maia P, Azevedo A, Amaral C, Tavares J. Stress and burnout among Portuguese anaesthesiologists. Eur J Anaesthesiol. 2006 May;23(5):433-9. https:// doi.org/10.1017/S0265021505001882.

23. Rui $M$, Ting $C$, Pengqian F, Xinqiao F. Burnout among anaesthetists in Chinese hospitals: a multicentre, cross-sectional survey in 6 provinces. J Eval Clin Pract. 2016 Jun;22(3):387-94. https://doi.org/10.1111/jep.12498.

24. Bland JM, Altman DG. Cronbach's alpha. BMJ (Clinical research ed). 1997 Feb 22;314(7080):572. https://doi.org/10.1136/bmj.314.7080.572.

25. Cronbach LJ. Coefficient alpha and the internal structure of tests. Psychometrika. 1951;16(3):297-334

26. Altman DG, Machin D, Bryant TN, Gardner MJ. Statistics with confidence. 2nd ed. Bristol: BMJ Books; 2000

27. Conover WJ. Practical non-parametric statistics. 3rd ed. New York: John Wiley; 1999.

28. Berenson M, Levin D, Szabat KA, Krehbiel TC. The Marascuilo procedure. Basic business statistics: concepts and applications. 12th ed. Frenchs Forest, NSW Australia: Pearson Australia; 2013. p. 575.

29. Marascuilo LA. Large-sample multiple comparisons. Psychol Bull. 1966 May:65(5):280-90. https://psycnet.apa.org/doi/10.1037/h0023189.

30. Ellis PD. The essential guide to effect sizes. New York: Cambridge University Press; 2010.

31. MedCalc Statistical Software. 18. 11th ed. Ostend, Belgium: MedCalc Software bvba; 2018.

32. Bryant T. Confidence Interval Analysis. 2.20 Build 57 ed: University of Southampton; 2011.

33. Sirsawy U, Steinberg WJ, Raubenheimer JE. Levels of burnout among registrars and medical officers working at Bloemfontein public healthcare facilities in 2013 S Afr Fam Pract. 2016;58(6):213-8. https://doi.org/10.1080/20786190.2016.1198 088

34. Weber A, Jaekel-Reinhard A. Burnout syndrome: a disease of modern societies? Occup Med (Lond). 2000 Sep;50(7):512-7. https://doi.org/10.1093/ occmed/50.7.512.

35. Lichtenstein RL. The job satisfaction and retention of physicians in organized settings: a literature review. Med Care Rev. 1984 Fall;41(3):139-79. https://doi. org/10.1093/occmed/50.7.512.

36. Freeborn DK. Satisfaction, commitment, and psychological well-being among HMO physicians. West J Med. 2001 Jan;174(1):13-8

37. De Oliveira GS Jr., Chang R, Fitzgerald PC, et al. The prevalence of burnout and depression and their association with adherence to safety and practice standards: a survey of United States anesthesiology trainees. Anesth Analg. 2013 Jul;117(1):182-93. https://doi.org/10.1213/ANE.0b013e3182917da9.

38. Dewa CS, Loong D, Bonato S, Trojanowski L. The relationship between physician burnout and quality of healthcare in terms of safety and acceptability: a systematic review. BMJ Open. 2017 Jun 21;7(6):e015141. https://doi.org/10.1136/ bmjopen-2016-015141.

39. Dewa CS, Loong D, Bonato $S$, Trojanowski $L$, Rea $M$. The relationship between resident burnout and safety-related and acceptability-related quality of healthcare: a systematic literature review. BMC Medical Education. 2017 Nov 9:17(1):195. https://doi.org/10.1186/s12909-017-1040-y.

40. Hall LH, Johnson J, Watt I, Tsipa A, O'Connor DB. Healthcare staff wellbeing burnout, and patient safety: a systematic review. PloS one. 2016;11(7):e0159015. https://doi.org/10.1371/journal.pone.0159015.

41. Panagioti M, Geraghty K, Johnson J, et al. Association between physician burnout and patient safety, professionalism, and patient satisfaction a systematic review and meta-analysis. JAMA Intern Med. 2018 Oct 1;178(10):1317-31. https://doi.org/10.1001/jamainternmed.2018.3713.

42. Williams ES, Manwell LB, Konrad TR, Linzer M. The relationship of organizational culture, stress, satisfaction, and burnout with physician-reported erro and suboptimal patient care: results from the MEMO study. Health Care Manage Rev. 2007 Jul-Sep;32(3):203-12. https://doi.org/10.1097/01. HMR.0000281626.28363.59.

43. Jones JW, Barge BN, Steffy BD, et al. Stress and medical malpractice organizational risk assessment and intervention. J Appl Psychol. 1988 Nov;73(4):727-35. https://psycnet.apa.org/doi/10.1037/0021-9010.73.4.727.

44. Honkonen T, Ahola K, Pertovaara M, et al. The association between burnout and physical illness in the general population-results from the Finnish Health 2000 Study. JPsychosom Res. 2006;61(1):59-66. https://doi.org/10.1016/j. jpsychores.2005.10.002.

45. Melamed S, Shirom A, Toker S, Berliner S, Shapira I. Burnout and risk of cardiovascular disease: evidence, possible causal paths, and promising research directions. Psychol Bull. 2006;132(3):327-353. https://doi. org/10.1037/0033-2909.132.3.327.

46. Ahola K, Gould R, Virtanen M, et al. Occupational burnout as a predictor of disability pension: a population-based cohort study. Occup Environ Med. 2009 May;66(5):284-90. https://doi.org/10.1136/oem.2008.038935. 
47. Kitaoka-Higashiguchi K, Morikawa Y, Miura K, et al. Burnout and risk factors for arteriosclerotic disease: follow-up study. J Occup Health. 2009:51(2):123-31. https://doi.org/10.1539/joh.L8104.

48. Ahola K, Väänänen A, Koskinen A, Kouvonen A, Shirom A. Burnout as a predictor of all-cause mortality among industrial employees: a 10-year prospective register-linkage study. J Psychosom Res. 2010 Jul;69(1):51-7. https://doi. org/10.1016/j.jpsychores.2010.01.002.

49. Toker S, Melamed S, Berliner S, Zeltser D, Shapira I. Burnout and risk of coronary heart disease: a prospective study of 8838 employees. Psychosom Med. 2012;74(8):840-7. https://doi.org/10.1097/PSY.0b013e31826c3174.

50. Frestad D, Prescott E. Vital exhaustion and coronary heart disease risk: a systematic review and meta-analysis. Psychosom Med. 2017;79(3):260-72. https://doi.org/10.1097/PSY.0000000000000423.

51. Toppinen-Tanner S, Ojajärvi A, Väänänen A, Kalimo R, Jäppinen P. Burnout as a predictor of medically certified sick-leave absences and their diagnosed causes. J Behav Med. 2005 Spring;31(1):18-32. https://doi.org/10.3200/BMED.31.1.18-32.

52. Bedi A, Courcy F, Paquet $M$, Harvey S. Interpersonal aggression and burnout: the mediating role of psychological climate. Stress Health. 2013;29(5):350-9. https:// doi.org/10.1002/smi.2476.

53. Bakker $A B$, Le Blanc PM, Schaufeli WB. Burnout contagion among intensive care nurses. J Adv Nurs. 2005;51(3):276-87. https://doi. org/10.1111/j.1365-2648.2005.03494.x.

54. Bakker AB, Schaufeli WB, Sixma HJ, Bosveld W. Burnout contagion among general practitioners. J Soc Clin Psychol. 2001;20(1):82-98. https://doi. org/10.1521/jscp.20.1.82.22251.

55. Dunford BB, Boss W, Boss AD, Zara G, Grooms R, editors. Can entire departments be burned out? A conservation of resources perspective on burnout contagion 2012: Academy of Management Briarcliff Manor, NY 10510. https://doi. org/10.5465/AMBPP.2012.12527abstract.

\section{Appendix}

\section{Definitions of the three dimensions of the burnout syndrome (verbatim quote from Maslach and Leiter) ${ }^{4}$}

"Emotional exhaustion refers to feelings of being overextended and depleted of one's emotional and physical resources. Workers feel drained and used up, without any source of replenishment. They lack enough energy to face another day or another person in need. The exhaustion component represents the basic individual stress dimension of burnout."

"Cynicism, also known as depersonalization, refers to a negative, hostile, or excessively detached response to the job, which often includes a loss of idealism. It usually develops in response to the overload of emotional exhaustion and is selfprotective at first - an emotional buffer of detached concern. But the risk is that the detachment can turn into dehumanization. The cynicism component represents the interpersonal dimension of burnout."

"Lack of Efficacy, also known as Personal Accomplishment, refers to a decline in feelings of competence and productivity at work. People experience a growing sense of inadequacy about their ability to do the job well, and this may result in a self-imposed verdict of failure. The inefficacy component represents the selfevaluation dimension of burnout."

Table A1: Interpretation of various statistical effect-sizes

\begin{tabular}{lll}
\hline Effect-size & Glass's $\boldsymbol{\Delta}$ & Probability of superiority \\
\hline None & 0 & 0.5 \\
Small & 0.2 & 0.56 \\
Medium & 0.5 & 0.64 \\
Large & 0.8 & 0.71 \\
\hline
\end{tabular}

Glass's delta gives the number of standard deviations of the control group by which the difference between two means differ. The probability of superiority is the probability that a person
56. Bianchi R, Schonfeld IS. Burnout-depression overlap: nomological network examination and factor-analytic approach. Scand J Psychol. 2018 Oct;59(5):5329. https://doi.org/10.1111/sjop.12460.

57. Bianchi R, Schonfeld IS, Laurent E. Burnout-depression overlap: a review. Clin Psychol Rev. 2015 Mar;36:28-41. https://doi.org/10.1016/j.cpr.2015.01.004.

58. Bianchi R, Schonfeld IS, Laurent E. Is it time to consider the "burnout syndrome" a distinct illness? Front Public Health. 2015;3:158. https://doi.org/10.3389/ fpubh.2015.00158.

59. Bianchi R, Schonfeld IS, Laurent E. Physician burnout is better conceptualised as depression. Lancet. 2017 Apr 8;389(10077):1397-8. https://doi.org/10.1016/ S0140-6736(17)30897-8.

60. Epstein RM, Privitera MR. Physician burnout is better conceptualised as depression - Authors' reply. Lancet. 2017 Apr 8;389(10077):1398. https://doi. org/10.1016/S0140-6736(17)30898-X.

61. Wallace JE, Lemaire JB, Ghali WA. Physician wellness: a missing quality indicator. Lancet. 2009 Nov 14;374(9702):1714-21. https://doi.org/10.1016/ S0140-6736(09)61424-0.

62. Ahola K, Kivimäki M, Honkonen $\mathrm{T}$, et al. Occupational burnout and medically certified sickness absence: a populationbased study of Finnish employees J Psychosom Res. 2008 Feb;64(2):185-93. https://doi.org/10.1016/j. jpsychores.2007.06.022.

63. Borritz M, Rugulies R, Christensen KB, Villadsen E, Kristensen TS. Burnout as a predictor of self-reported sickness absence among human service workers: prospective findings from three year follow up of the PUMA study. Occup Environ Med. 2006 Feb;63(2):98-106. https://doi.org/10.1136/oem.2004.019364.

64. Grissom RJ. Probability of the superior outcome of one treatment over another. J Appl Psychol. 1994 Apr;79(2):314-6. https://psycnet.apa.org/ doi/10.1037/0021-9010.79.2.314.

65. Sheskin D. Probability of superiority as a measure of effect size. Handbook of parametric and nonparametric statistical procedures. 4th ed: Chapman \& Hall; 2007. p. 801

picked at random from one group will have a higher score than a person picked at random from the other group.

Table A2: Calculation of the effect-sizes of various tests

\begin{tabular}{lccc}
\hline $\begin{array}{l}\text { Difference between } \\
\text { point estimates }\end{array}$ & $\begin{array}{c}\text { Effect-size } \\
\text { index }\end{array}$ & Equation & Reference \\
\hline $\begin{array}{l}\text { Means } \\
\text { (t-test) }\end{array}$ & Glass's delta & $\Delta=\frac{\mathrm{m}_{1}, \mathrm{~m}_{2}}{\mathrm{~s}_{\mathrm{c}}}$ & $\begin{array}{c}\text { Ellis } \\
(2010)^{31}\end{array}$ \\
$\begin{array}{l}\text { Medians } \\
\text { (Mann-Whitney U) }\end{array}$ & $\begin{array}{c}\text { Probability of } \\
\text { superiority }\end{array}$ & $P S=\frac{\mathrm{U}}{\mathrm{n}_{1}, \mathrm{n}_{2}}$ & $\begin{array}{c}\text { Grissom } \\
(1994)^{65}\end{array}$ \\
$\begin{array}{lccc}\text { Medians } \\
\text { (Wilcoxon signed rank) }\end{array}$ & $\begin{array}{c}\text { Probability of } \\
\text { superiority }\end{array}$ & $P S=\frac{\mathrm{n}_{+}}{\mathrm{N}}$ & $\begin{array}{c}\text { Sheskin } \\
(2007)^{66}\end{array}$ \\
\hline
\end{tabular}

$m_{1} m_{2}-$ means of first and second groups; $s_{c}-$ standard deviation of control group

$\mathrm{n}_{1}, \mathrm{n}_{2}-$ numbers of subjects in the two groups; $\mathrm{N}-$ total sample size

$n-$-number of positive differin

U-Mann-Whitney U statistic;

Probability of superiority (PS) is also known as the common language effect-size statistic,

probabilistic index, intuitive and meaningful effect-size index, and the measure of stochastic superiority

\section{The margin of error of a survey's random sample:}

The "margin of error" of a survey is an estimate of its general precision and it is dependent on the sample size. It is defined as the widest possible radius of the confidence interval of a particular statistic obtained from the survey. This is perhaps best explained as follows. Suppose that a proportion of participants, $p$, from a random sample of size, $n$, return a particular answer to a certain question. The confidence interval for that proportion is given by $p \pm Z_{\alpha / 2}$ times the standard error of $p$, where $Z_{\alpha / 2}$ is the desired degree of confidence. Thus

Equation $\mathrm{A} 1$ :

Confidence interval $=\mathrm{p} \pm \mathrm{Z}_{\alpha / 2} * \sqrt{\frac{\mathrm{p} *(1-\mathrm{p})}{\mathrm{n}}}$ 
For confidence intervals of $90 \%, 95 \%$ and $99 \%$ values for $Z_{\alpha / 2}$ are $1.645,1.96$ and 2.58 respectively. The radius of a confidence interval is half its width, therefore the radius is given by

Equation A2:

Radius $=\mathrm{Z}_{\alpha / 2} * \sqrt{\frac{\mathrm{p} *(1-\mathrm{p})}{\mathrm{n}}}$

Figure $A 1$ depicts the radii of the $95 \%$ confidence intervals of samples of various sizes, versus $p$, the proportion of the sample. It can be seen that the maximum radius of a sample proportion occurs at a proportion of 0.5 and is smallest for proportions close to 0 and 1. The margin of error is defined as the maximum radius and is depicted by the dotted line, i.e. at a proportion of 0.5 . Note that the margin of error decreases as the sample size increases.

Figure A1: Radii of $95 \%$ confidence intervals for variously sized samples

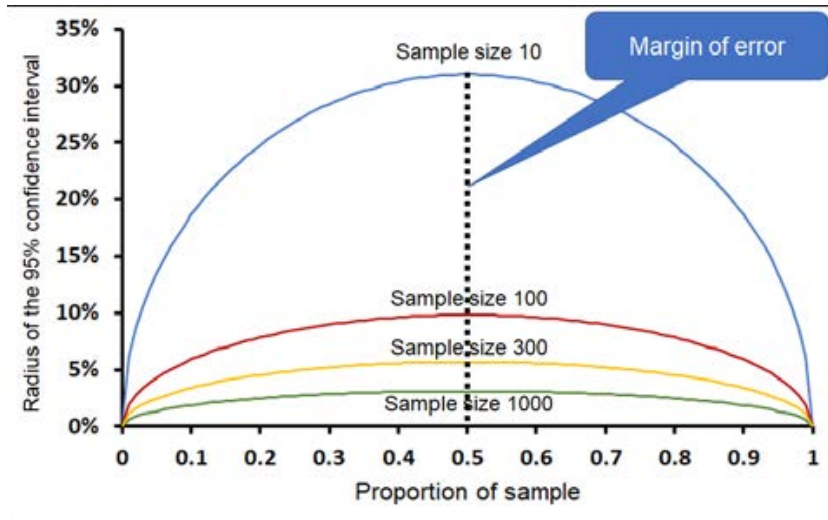

A small correction can be made for a finite population size:

Equation A3:
Finite Population Correction $=\sqrt{\frac{N-n}{N-1}}$

Where $\mathrm{N}$ is the population size.

Table A3 displays various margins of errors for different sample sizes, corrected for a population size of 1852 .

\begin{tabular}{cc} 
Table A3 & \\
\hline Sample size & Margin of error \\
\hline 10 & $31 \%$ \\
100 & $9.5 \%$ \\
300 & $5.2 \%$ \\
500 & $3.75 \%$ \\
1000 & $2.1 \%$ \\
\hline
\end{tabular}

\section{Calculation of the required sample size for a survey}

The desired margin of error is used to calculate a priori the sample size for a planned survey.

Equation A4:

Sample size $=\frac{\frac{\mathrm{z}^{2} * \mathrm{p} *(1-\mathrm{p})}{\mathrm{err}^{2}}}{1+\left(\frac{\mathrm{z}^{2} * \mathrm{p} *(1-\mathrm{p})}{\mathrm{err}^{2} * \mathrm{~N}}\right)}$

Where $z=Z_{\alpha / 2}$ (1.96 for a 95\% confidence interval); $p=a$ proportion of 0.5 ; err $=$ desired margin of error; $N=$ population size.

There are various Internet websites that enable calculations of margins of error and sample sizes for surveys, for example https://www.checkmarket.com/sample-size-calculator/ and https://www.surveymonkey.com/mp/sample-size-calculator/. 